\title{
A projection-based model reduction method for nonlinear mechanics with internal variables: application to thermo-hydro-mechanical systems.
}

\author{
Angelo Iollo ${ }^{1,2}$, Giulia Sambataro ${ }^{1,2}$, and Tommaso Taddei ${ }^{1,2}$ \\ ${ }^{1}$ Memphis Team \\ INRIA Bordeaux Sud-Ouest \\ Talence, France \\ ${ }^{2}$ Institut de Mathématiques de Bordeaux \\ Université de Bordeaux \\ Talence, France \\ E-mail: angelo.iollo@inria.fr, giulia.sambataro@inria.fr, tommaso.taddei@inria.fr
}

Key Words: THM systems; parametrized partial differential equations; model order reduction; nonlinear mechanics.

\begin{abstract}
We propose a projection-based monolithic model order reduction (MOR) procedure for a class of problems in nonlinear mechanics with internal variables. The work is is motivated by applications to thermo-hydro-mechanical (THM) systems for radioactive waste disposal. THM equations model the behaviour of temperature, pore water pressure and solid displacement in the neighborhood of geological repositories, which contain radioactive waste and are responsible for a significant thermal flux towards the Earth's surface. We develop an adaptive sampling strategy based on POD-Greedy method, and we develop an elementwise empirical quadrature hyper-reduction procedure to reduce assembling costs. We present numerical results for a two-dimensional THM system to illustrate and validate the proposed methodology.
\end{abstract}

\section{References}

[1] Reduced basis methods: success, limitations and future challenges. M. Ohlberger and S. Rave. In arXiv preprint arXiv:1511.02021. 2015.

[2] A priori hyper-reduction method for coupled viscoelastic-viscoplastic composites. D. Ryckelynck et al. In Journal of computational physics. Elsevier. 2013.

[3] Building a certified reduced basis for coupled thermo-hydro-mechanical systems with goal-oriented error estimation. Larion Y. et al. In Springer Nature. 2020 .

[4] Structure-preserving, stability, and accuracy properties of the energyconserving sampling and weighting method for the hyper reduction of nonlinear finite element dynamic models. Farhat et al. In International Journal for Numerical Methods in Engineering. Wiley Online Library. 2015. 